\title{
La colonización de sexualidades indígenas: entre despojo y resistencia
}

Manuela L. Picq'

Resumo: La colonización sexual reprime sexualidades indígenas, regulando las experiencias sexuales y de género, forzando pueblos indígenas a existir dentro de códigos occidentales heteronormativos. Este ensayo analiza la supresión de las sexualidades indígenas como parte de un proceso más amplio de despojo colonial. Se examina la diversidad sexual en lenguas indígenas, la narrativa colonial de sodomía que criminaliza cuerpos para justificar el despojo bajo la doctrina del descubrimiento, e se propone descolonizar las sexualidades como un acto fundamental de libre determinación para resistir el despojo en todas sus formas.

Palabras llave: sexualidad, genero, pueblos indígenas, colonialismo, sodomía.

The colonization of indigenous sexualities: between dispossession and resistance

Abstract: Sexual colonization represses indigenous sexualities, regulating sexual and gender experiences, forcing indigenous peoples to exist within western heteronormative frameworks. This essay analyzes the erasure of indigenous sexualities as part of a broader process of colonial dispossession. It examines sexual diversity in indigenous languages, the colonial narrative of sodomy that criminalizes bodies to justify dispossession under the doctrine of discovery, and proposes to

$1 \quad$ Universidad San Francisco de Quito-USFQ - Quito - Ecuador - y Amherst College (EUA) - mpicq@ amherst.edu 
decolonize sexualities as a fundamental act of self-determination to resist ongoing forms of dispossession.

Keywords: sexuality, gender, indigenous peoples, colonialism, sodomy.

Los nahuas de Tenochtitlan no tenían la palabra sexo. Tenían sexo, por supuesto, de todo tipo, pero su idioma no tenía una categoría traducible como "sexo" o "sexualidad". El náhuatl utilizaba el concepto de xochihua (portador de flores) para expresar las conexiones íntimas con los demás y la fertilidad en todas sus formas (Sigal, 2011: 2). ${ }^{2}$ Las flores representan el sexo en las construcciones nahuas del mundo: son la metáfora de todas las fuerzas de la vida y se asocian con la fertilidad, la filosofía y la poesía. Pero los misioneros españoles malinterpretaron las cosmovisiones nahuas cuando escribieron el Códice Florentino, una crónica del siglo XVI sobre la vida nahua. Ellos tradujeron $x o-$ chihua como sodomita (Sigal, 2011), transformando una metáfora de vida en una noción negativa de pecado. Los nahuas no equiparaban el sexo con pecado; visualizaban las relaciones sexuales como elementos de un conjunto mucho más amplio de prácticas rituales para promover la fertilidad de los dioses, los humanos y de la tierra (Sigal, 2011). La traducción al español no solo distorsionó la visión nahua del mundo; en el proceso, también la invisibilizó.

Siglos después, otras lenguas indígenas se enfrentan a nuevas distorsiones, con riesgos similares. En tikuna, un idioma aislado de la Amazonia sin ninguna ascendencia común o genealogía demostrable con otro idioma, la palabra Kaigüwecü describe a un hombre que tiene relaciones sexuales con otro hombre y Ngüe Tügümaêgüé a una mujer que tiene relaciones sexuales con otra mujer (Tikuna; Picq, 2016). Hoy en día, estas palabras se entienden dentro de los marcos LGBT como evidencia de que los géneros binarios occidentales no son ni universales ni verdades biológicas. ¿Pero, se puede traducir Ngüe Tügümaêgüé como lesbiana? Josi Tikuna rechaza lésbica como traducción, diciendo que esta palabra introducida por misioneros implica connotaciones negativas de pecado y no toma en cuenta visiones tikunas del mundo. ¿Qué mundos se pierden cuando reducimos relacionalidades indígenas a codificaciones LGBT? El reto no es solo la imposibilidad de explicar mundos

2 El náhuatl se habla desde el siglo VII en el centro de México y fue el idioma de los aztecas. Durante la invasión española, el náhuatl fue utilizado para códices, crónicas, documentos administrativos y poesía, convirtiéndose en una de las lenguas precolombinas mejor documentadas en todo el continente. Hoy, casi dos millones de personas hablan variaciones de náhuatl en comunidades dispersas en Mesoamérica. 
indígenas en términos eurocéntricos, sino también el riesgo de despojo que acompaña traducciones inadecuadas.

Las sexualidades indígenas son intraducibles en términos occidentales. No son las palabras las que son intraducibles, sino el tejido social, cultural y político que representan. Las taxonomías desarrolladas por la sociedad colonial no pudieron capturar las prácticas nahuas en el siglo XVI así como los marcos actuales de derechos sexuales no pueden capturar las relaciones tikunas. Tanto los curas españoles como los activistas LGBT sólo pueden entender los mundos indígenas filtrados a través de sus propias nociones de la sexualidad (Cottet; Picq, 2019). Esto plantea un problema epistemológico; también plantea un problema de despojo, ya que la traducción forzada se convierte en una forma de apropiación política.

El proyecto heteronormativo ha sido analizado como un proyecto de modernidad europea ligado al Estado-nación. La concepción binaria de género se convirtió en un indicador de la sexualidad moderna, y la heterosexualidad como un principio clave de la construcción de nuevos estados-nación. Afsaneh Najmabadi investigó la heterosocialización del espacio público y la reconfiguración de la vida familiar en el Irán del siglo XIX: las prácticas homosexuales comenzaron a señalar atraso político, y la heteronormalización del eros se convirtió en una condición para "alcanzar la modernidad" (Najmabadi, 2005: 3). La formación de la modernidad iraní produjo binarios de género, en gran parte a través de la eliminación de las formas de vida repentinamente redefinidas como atrasadas. En la India del siglo XIX, las autoridades coloniales británicas crearon una ley antisodomía que reflejaba sus valores cristianos para criminalizar las prácticas sexuales consideradas "contra el orden de la naturaleza" (Puri, 2016). En 1860, esta ley se introdujo en el Código Penal de la India como Sección 377, luego se extendió en 40 países de todo el Imperio Británico, desde Malasia hasta Uganda, y en muchos casos siguió existiendo mismo después que declaraciones de independencia terminen con el régimen colonial (Human Rights Watch, 2008: 6). Las leyes sobre sexualidad permitieron, sostuvieron y produjeron sujetos nacionales en términos de género en la vida cotidiana. Determinaron lo que era natural y lo que no lo era para definir lo que era legal e ilegal. Estas leyes se utilizaron para controlar y reprimir todo tipo de transgresión, y aun se utilizan en el sureste asiático en acusaciones de "antinacionalismo", "sedición" o "blasfemia" (Svati, 2019: 1).

Aun que las reglas de la heteronormatividad afectaron todos los pueblos colonizados, su resonancia fue distintiva en la colonización del llamado Nuevo Mundo. Allí, se inscribía como parte de un profundo despojo territorial. En la Abya Yala, nombre que los pueblos indígenas utilizan para referirse al 
hemisferio occidental, la heterosexualización era un elemento mas en la doctrina del descubrimiento. La sexualidad fue una herramienta para criminalizar a los nativos, enmarcarlos como pervertidos y validar la violencia europea contra el "otro" no cristiano, etiquetado como salvaje, hereje y sodomita. Disciplinar los cuerpos nativos fue un eje central del proceso colonial. En esta investigación, se explora el papel de la heteronormatividad en la colonización sexual para mostrar como el despojo es un fenómeno multifacético y estructural que afecta a todo tipo de territorios, incluido el cuerpo.

Esta investigación aborda las sexualidades indígenas no para ilustrar la adaptación indígena a la diversidad sexual ni para proponer un cosmopolitismo indígena. Las visiones indígenas sobre el rol de la sexualidad son específicas a cada cultura. El discurso internacional sobre derechos sexuales no introdujo referentes para reconocer sexualidades alternativas; las lenguas indígenas ya las tenían, como lo indican sus terminologías. Las sexualidades indígenas importan por lo que podemos aprender de ellas, no sobre ellas (Fernandes; Arisi, 2017). Ellas nos permiten entender lo profundo que fue el despojo al revelar la heteronormatividad como instrumento de control y destrucción colonial (Simpson, 2017). Contribuyen a los debates poscoloniales sobre el despojo colonial, la criminalización de las sexualidades no europeas y el control (siempre incompleto) de los cuerpos, deseos e intimidades colonizados. El colonialismo busca interrumpir las relaciones políticas, e por ente íntimas, de los pueblos indígenas. Por eso los académicos insisten simultáneamente en el valor de descolonizar los estudios queer y los estudios queer descoloniales (Driskill et al., 2011; Morgensen, 2011). Kim TallBear (2018: 148), una intelectual indígena del pueblo Dakota Sisseton Wahpeton Oyate, insiste en la importancia de reconocer las intimidades indígenas para descolonizar el sexo y formas de relacionarnos. Las experiencias indígenas son valiosas porque redefinen la pertenencia y las relaciones, porque amplían epistemologías y reverter ontologías. Las experiencias indígenas confirman que las narrativas actuales de naturalización de la familia patriarcal heteronormativa son un fenómeno moderno importado de Europa que debe ser contextualizado.

Quiero aclarar con Gayatri Spivak (1993) sobre la necesidad e imposibilidad de la traducción. Los significados de los roles de género y las prácticas sexuales son construcciones culturales que inevitablemente se pierden cuando se descontextualizan en la traducción cultural (y lingüística) (Cottet; Picq, 2019). Los archivos de sodomía de la época colonial están incrustados en nociones de sexo e interpretaciones del cuerpo propios de la época. Hablar de sexualidades indígenas en la lengua del colonizador corre el riesgo permanente de anacronismo y tergiversación. 
La idea de que una persona es homosexual, por ejemplo, surge de las suposiciones contemporáneas de identidad sexual y sólo es posible después de la invención de la homosexualidad (Katz, 2007). Mark Rifkin (2011) pregunta cuándo el indio se convirtió en heterosexual porque el vocabulario heterosexual es tan inapropiado como la imaginación binaria para entender los mundos indígenas. Las normas internacionales de derechos sexuales no pueden dar cuenta del deseo, de la vida o de la pertenencia en mundos precolombinos. Este estudio se inscribe en estas imposibilidades y peligros de la traducción epistemológica.

También quiero aclarar la terminología. Utilizo varios términos para referirme a los pueblos indígenas por que diferentes experiencias coloniales resultaron de una pluralidad de relaciones de poder. El concepto de indio surgió en el siglo XVI como una categoría colonial que se refería a los pueblos no europeos desde las Indias del Este al Oeste, todos construidos como una otredad de Europa (Seth, 2010). Crear una categoría jurídica que homogeniza a tantos pueblos distintos es un acto de dominio colonial (Van Deusen, 2015). Las definiciones oficiales de quien es indígena varían dependiendo de cuando y como los estados manipulan leyes, censos y hasta la ciencia para borrar, aumentar o desplazar la presencia indígena de acuerdo con sus intereses económicos (Kauanui, 2008). Los pueblos indígenas son 370 millones de individuos en noventa países; más de cinco mil naciones que hablan miles de idiomas en diferentes sistemas culturales y espirituales. Les caracteriza la diversidad, e si tienen algo en común es una historia de colonización europea.

Este ensayo muestra cómo la colonización sexual reprime sexualidades indígenas, regulando las experiencias sexuales y de género, forzando pueblos indígenas a existir dentro de los códigos occidentales heteronormativos, que sea adoptando principios cristianos heterosexuales o imponiendo códigos LGBT. Se analiza la supresión de las sexualidades indígenas como parte de un proceso más amplio de despojo colonial. Una primera sección examina la diversidad sexual que sobrevive en las lenguas indígenas. Una segunda sección explica la narrativa colonial de sodomía para criminalizar los cuerpos indígenas y justificar la conquista territorial bajo la doctrina del descubrimiento. Una tercera sección argumenta que descolonizar las sexualidades indígenas es un acto fundamental de libre determinación para resistir el despojo en todas sus formas.

\section{Sexos e géneros antes de la colonia}

Antes de la invasión europea, la sexualidad era un elemento vital que no era ni asociado a pecado, ni regulado, e menos criminalizado. Los pueblos de Abya 
Yala celebraban sexualidades diversas en tiempos precolombinos. La cerámica Moche (15-80o d.C.) en la costa norte del Pacífico del actual Perú celebra la sexualidad como fuerza vital. Los recipientes de estribo Moche representan una variedad actos sexuales, pero raramente la penetración vaginal, enfatizando los genitales masculinos y el movimiento de fluidos entre los cuerpos como una forma de comunicación (Weismantel, 2004). Al otro lado del Pacífico, las esculturas Māori celebraban relaciones sexuales entre personas del mismo sexo y entre cuerpos múltiples (Te Awekotuku, 2005). En los Andes, los Inkas convocaron a una figura extraña llamada chuqui chinchay para mediar en una crisis política a finales del siglo XV (Horswell, 2005). El chuqui chinchay, una figura venerada en la cultura andina era la deidad de la montaña de los jaguares e el patrón de personas de doble género. Encarnaban una fuerza creativa que combinaba lo masculino y lo femenino en el dualismo de la filosofía andina y realiza rituales shamánicos, incluso con prácticas eróticas del mismo sexo.

A los colonizadores les costaba reconocer estas sexualidades. Las crónicas coloniales describían sexualidades que no lograban ni entender y menos aceptar. Will Roscoe (1998: 12-15) reunió documentos coloniales que informaban sobre géneros alternativos. Las expediciones francesas en Florida describieron "hermafroditas" entre los pueblos Timucua ya en 1564. Los grabados coloniales los describen como guerreros, cazadores y tejedores. En el Valle del Mississippi, los colonizadores franceses reportaron un tercer género llamado ikoueta en el idioma algonquino, varones que adoptaron otros roles de género. Eran guerreros, shamanes en ceremonias y lideraban consejos de sabios. Según los informes coloniales, eran seres superiores, y nada podía decidirse sin su consejo. Otro colonizador francés, Dumont de Montigny, describió a los varones que hacían el trabajo de las mujeres y tenían relaciones sexuales con los hombres entre los Natchez en el bajo Mississippi en el siglo XVIII. En lo que hoy es Texas, el colonizador español Cabeza de Vaca reportó a hombres que se vestían y que vivían como mujeres. En el Ártico, el pueblo Inuit también platicaba sexualidades flexibles y diversas, como lo describe el colonizador Saladin d'Anglure. En la región subártica, los rusos reportaron fallar en sus intentos de suprimir las cosmovisiones Chugach y Koniag que celebraban a quienes eran "dos personas en una" y los consideraron afortunados.

Roscoe (1998) coleccionó mas de 150 taxonomías documentando diversas percepciones indígenas de género. En el idioma del pueblo Diné, también conocido como Navajo, nádleehí significa "quien cambia", e los pueblos Osage, Omaha, Kansa y Oto usan el término mixu'ga para expresar "instruido por la luna", refiriéndose a las distintas habilidades e identidades que la luna brinda 
a ciertas personas (Roscoe, 1998: 13). En muchas culturas, tener varios géneros se asociaba con poderes espirituales. En la cosmovisión Lakota, las personas winkte tenían poderes auspiciosos y podían predecir el futuro; los guerreros les visitaban antes de ir a la batalla para adquirir mas fuerza. En la cosmovisión Cheyenne, personas he'emane'o encarnaban los principios centrales de equilibrio y síntesis, e eran quienes dirigían la importante danza de la victoria (Roscoe, 1998: 14).

La gran parte de los archivos coloniales documenta solamente desviaciones del género masculino, probablemente porque era lo que más les horrorizaba y porque juzgaban a las mujeres irrelevantes. Pero las mujeres también se dedicaban a prácticas del mismo sexo y a géneros alternativos que marcaban roles en sus comunidades. Casi un tercio de los grupos del índice lingüístico de Roscoe se refiere específicamente a mujeres que asumían roles masculinos. Evelyn Blackwood (1984) explica que la homosexualidad constituía una oportunidad para que las mujeres asumieran roles masculinos de manera permanente y se casaran con mujeres. Un ejemplo famoso es quizás la icónica Woman Chief (mujer cacique), una líder del pueblo Cree que estuvo al frente de la resistencia armada contra los invasores europeos. Un comerciante de la Compañía Americana de Pieles que viajaba por el río Missouri documenta en sus crónicas que Woman Chief no solo era líder militar de su pueblo, pero también tenía cuatro esposas y era una de las autoridades principales en el Concejo de Autoridades Cree (Roscoe, 1998: 78).

Pero ¿cómo pensar estas expresiones diversas de géneros a través de metáforas o palabras intraducibles? Blackwood (1984:35) argumenta que los pueblos indígenas de Norte América disociaban el sexo del genero: la sexualidad no determinaba el género - los individuos tenían una identidad de género, pero no una identidad sexual correspondiente. En otras palabras, una mujer no solamente podía tener relaciones eróticas con otra mujer, sino que esto no determinaba su identidad de género, ni hacia que ella sea considerada "lesbiana". Es decir que el sexo no estaba enredado en practicas de género como lo es hoy. Blackwood enfatiza la poca importancia del sexo biológico para los roles de género en las visiones indígenas del mundo. Había mucha superposición entre lo masculino y lo femenino, y las personas que una vez estuvieron casadas y tuvieron hijos, más tarde en la vida buscaban relaciones del mismo sexo. Roscoe (1998: 10) interpreta esta fluidez como una distinción entre sexo reproductivo y el sexo erótico no-reproductivo, contrario a la distinción actual entre homo/ hetero-sexualidad. Las interpretaciones varían, y los géneros y sexualidades indígenas varían inmensamente según contextos sociales, culturales y políticos 
en cada pueblo. Lo que es seguro es que las sociedades indígenas han respetado, integrado y a incluso admirado sexualidades no heterosexuales y géneros no-binarios durante mucho tiempo.

Los archivos históricos y lingüísticos son fuentes importantes pero la traducción queda corta: son palabras que se refieren a tejidos sociales que han sido ampliamente despojados, reprimidos y destruidos. Cada idioma indígena aporta una comprensión singular del género. Los géneros indígenas no pueden reducirse a nociones de heterosexualidad, homosexualidad o transexualidad. Sería un anacronismo, y hasta una forma de despojo, traducir estos tejidos sociales precolombinos a marcos contemporáneos de derechos LGBT. En las sociedades de pre-conquista, los géneros no-binarios e las sexualidades no heteronormativas no eran una anomalía o diferencia, sino que eran constitutivas de un todo. El debate de como traducir estas realidades es casi irrelevante. El valor de estudiar sexualidades indígenas no es para añadir más géneros a los registros sexuales establecidos; ellas invocan tejidos sociales complejos que son intraducibles en el marco limitado de la hetero/homosexualidad. Invocan epistemologías y visiones del mundo indígena, más allá del cuerpo y del placer.

Los colonizadores europeos no alcanzaron (o no quisieron) ver conceptualizaciones del mundo distintas de las suyas. Vanita Seth (2010) explica que la dificultad europea para reconocer la diferencia proviene de una incapacidad más amplia de traducir el Nuevo Mundo al lenguaje político familiar. Tanto entonces como ahora, la capacidad de reconocer otras formas de conocimiento está entrelazada con las formulaciones del ser político. "Es difícil hablar el lenguaje de la alteridad cuando el otro está virtualmente ausente del discurso del yo" (Seth, 2010: 38). El "descubrimiento" se vio gravemente perjudicado por la incapacidad de los colonizadores de convertir lo que encontraron en el Nuevo Mundo en un lenguaje accesible a si mismos.

El desafío persiste. Las sexualidades indígenas todavía resisten a la traducción y categorización sexual. Para el pueblo Hawái, los māhū son los que están en el medio (Wilson; Hamer, 2014). Los hawaianos ven el género como un continuo, y desde tiempos ancestrales reconocen que algunas personas no son simplemente femeninas o masculinas (Robertson, 1989; Tengan, 2008). El ser $m \bar{h} \bar{u}$ abarca tanto los rasgos femeninos como los masculinos. Como los winkte del pueblo Lakota, han sido históricamente respetados como cuidadores y sanadores que transmiten conocimiento. Aun son valorados hoy al encarnar los principios polinesios de dualidad e integración espiritual, la síntesis femenina/masculina en la filosofía hawaiana. Algo parecido pasa en Juchitán, México, donde las muxes se niegan a ser traducidos como hombres que se visten como 
mujeres (Mirandé, 2017). La sociedad zapoteca de Juchitán se caracteriza por mujeres extraordinariamente fuertes y autónomas que ocupan cargos políticos, y muchos la describen como una sociedad que goza de igualdad de género. Las muxes, que no se definen ni como hombres ni mujeres sino su proprio género, fueron tradicionalmente vistas como una bendición de los dioses; hoy en día siguen siendo una parte integral de la sociedad, socialmente aceptadas y plenamente activas con responsabilidades familiares y comunitarias.

El ser māhù y muxes no puede reducirse dentro de las categorizaciones LGBT, ni su experiencia puede ser exportada o replicada en otros lugares. Estas identidades existen en sus propios contextos e idiomas, son imposibles de trasladar. Dicen los abuelos que el antiguo idioma zapoteco no diferenciaba el hombre e la mujer. En zapoteco, la-ave se refería a la gente, la-ame a los animales, y la-ani a los seres inanimados (Olita, 2017). Fueron los españoles quienes introdujeron los géneros femenino y masculino. ¿Cómo traducir muxes en idiomas del zapoteco al español que se estructura en torno al género? Son muchos registros intraducibles. ¿Cuáles son las implicaciones de traducir las sexualidades periféricas en el idioma del centro? La traducción violenta de las formas de vida en la codificación legal marcó siglos de despojo colonial, y el desafío sigue tan relevante en la actualidad.

\section{2. "Todos sodomitas": sexualidad y doctrina del descubrimiento}

Los colonizadores no entendieron nada de las sexualidades con las cuales se toparon en el Nuevo Mundo. Así que tradujeron las prácticas locales en sus términos europeos y cristianos, criminalizando la alteridad al enmarcar las sexualidades nativas como perversas y antinaturales. La sodomia era tema constante en sus crónicas, mismo cuando el cronista nunca había dejado Europa como lo hizo Francisco López de Gomora, quien escribió la Historia General de las Indias, sin viajar a América. Los relatos describen a los indios como "grandes sodomitas" que "realizan actos carnales con hombres y mujeres sin vergüenza" (Mirandé, 2017: 53). En 1540, Cabeza de Vaca describe en al pueblo Karankawa (en el actual Texas) como bestial por que "un hombre estaba casado con otro y (...) hombres impotentes que se visten como mujeres" (Roscoe, 1998: 4). En América del Norte, los colonizadores franceses definen a los ikouetas con referencias a "sodomía" (Roscoe, 1998: 13) y Loskiel acusa al pueblo Lenape de "crímenes contra natura" (Roscoe, 1998: 251). El mismo concepto se repetia en los Andes. Crónicas como la Relación de Servicios en Indias, calificaron al chuqui chinchay como diabólico y declara que los indios "son todos sodomitas" 
(Horsewell, 2005: 1-2). Los españoles veían las sexualidades de los Inkas como abyectas; los dioses extraños no respetaban las reglas, jerarquías y fronteras de género como la cultura cristiana española. Tras definirlos como sodomitas, llamaron a su exterminio.

Las frecuentes referencias a sodomitas en archivos coloniales van de mano con una brutal represión de las personas acusadas de prácticas contra natura en todo el continente. Un ejemplo infame es la masacre de supuestos "sodomitas" en 1513 por el conquistador español Vasco Núñez de Balboa, en Panamá. Balboa arrojó al hermano del cacique Quaraca y a cuarenta de sus compañeros a los perros por estar supuestamente vestidos de mujer. La masacre es contada en Les Grands Voyages, de Theodore de Bry en 1594. En 1536, Frey Bartolomé de las Casas reporta la masacre de tres hombres por estar vestidos de mujer (Carillo Can, 2015: 79). Los españoles determinaban el pecado (de sodomía) y arrojaron seres humanos a los perros. En otro episodio macabro, los colonizadores franceses matan a un hombre en el norte de Brasil por ser "más hombre que mujer" y "para purificar la tierra" (Fernandes; Arisi, 2017: 7). Los asesinos europeos ataron esta persona a la boca del cañón y obligaron que su propio cacique encendiera la mecha que desmantelaba el cuerpo en partes delante de todos los demás "salvajes". El hecho de etiquetar a las poblaciones nativas como sodomitas justificaba, en la mirada de los colonizadores, masacre y despojo para "salvar" la tierra.

La narrativa de la conquista enfatizaba los ideales europeos de género que describían a los invasores como masculinos, racionales, poderosos y a las poblaciones indígenas como femeninas, irracionales y pecaminosas (Molina, 2017; Sigal, 2011; Trexler, 1999). Las nociones de sodomitas estaban entrelazadas en las percepciones europeas de masculinidad, y ambas sirvieron para organizar el poder en el escenario colonial (Carvajal, 2003). Pero el tema no era simplemente feminizar al enemigo; se invocaba a los sodomitas a propósito. Las sexualidades indígenas eran desaparecidas de la manera mas brutal. Los colonizadores acusaban a los pueblos del continente de sodomía sin mismo tener claro lo que era, por que a la final no se referían a un acto específico, sino que a cualquier práctica considerada fuera de los códigos europeos de conducta católica (Horswell, 2005: 15).

Mark Jordan (1997) atribuye al teólogo Peter Damian el haber acuñado la palabra sodomía por analogía con la blasfemia en el siglo XI. En su primera iteración, la sodomía se refiere al pecado explícito de desobedecer a Dios en el Antiguo Testamento (Jordan, 1997: 29). La historia de Sodoma es una historia de castigo divino para quienes desafían la autoridad divina: es un pecado de (des) obediencia, no de (homo)sexualidad. Inicialmente, la palabra sodomía se refiere a la herejía: algunos de los primeros sacerdotes acusados de idolatría también 
fueron acusados del crimen de Sodoma (Jordan, 1997: 36). Los primeros sodomitas eran herejes, muchos eran mujeres. La noción de sodomía como pecado sexual contra natura surge con Tomás de Aquinas. Su Summa Theologiae (cerca de 1270) fue utilizada por el Concilio de Trento para su legislación doctrinal; ese documento crea los pecados de la lujuria como pecados carnales que se manifiestan en el exceso de placeres. Aquinas define seis tipos de pecado: fornicación, adulterio, incesto, desfloración, secuestro y vicio contra natura.

La sodomía evoluciona desde la blasfemia para significar placeres carnales contra natura, siempre de forma ambigua, y por ente permitiendo su uso arbitrario. Lo importante es entender que la sodomía empieza a demarcar el límite entre el orden natural (tal como lo define Dios) y el otro salvaje, separando lo legitimo de lo ilegitimo, lo legal de lo ilegal, y convirtiéndose en una herramienta para las invasiones europeas y el despojo. Los colonizadores españoles lo usaron como parte del léxico de la empresa colonial. Los crímenes contra natura crearon dicotomías que demuestran la esencia reproductiva del proyecto colonial: natural / antinatural; reproductivo / sodomita, humano / animal. Quienes eran definidos como sodomitas eran castigados con muerte y sus bienes eran apropiados por los españoles. En 1601, por ejemplo, dos hombres indígenas de Chiquimula son "condenados a la hoguera" por sodomía y todos sus bienes son confiscados (Tortorici, 2018: 74). La sodomía invocaba la autoridad divina para justificar la destrucción europea de los mundos indígenas (Fernandes; Arisi, 2017). Hawkins (2012) explica la noción de Sodoma del Nuevo Mundo como una estrategia de conquista ibérica del siglo XVI que comparaba a los nativos del Nuevo Mundo con los habitantes de Sodoma y Gomorra a través de herramientas literarias como la intertextualidad, la alusión, la imitación y la parodia.

La sodomía fue buena herramienta de colonización precisamente porque era ambigua. Se refería a todo lo que contravenía los códigos de autoridad aceptados por la Iglesia. Los colonizadores la invocaban para reprimir cualquier práctica que no se ajustara a su regla. Esto quedó claro durante el Debate de Valladolid de 1550, cuando los teólogos españoles debatieron si los indios tenían alma y si podían ser esclavizados. Juan Ginés de Sepúlveda argumentó que los pueblos indígenas de las Américas no tenían alma, y equiparó su estatus moral al de las mujeres para justificar la tutela. Sepúlveda sugirió que los europeos debían cumplir con el papel del Señor, haciendo legal el someterlos al dominio español. No eran sus prácticas sexuales las que estaban en juego, sino la apropiación de sus tierras. La descripción de los pueblos indígenas siendo "todos sodomitas" era una herramienta más para justificar el despojo, las masacres, y la invasión de los territorios. 
Lo que quiero decir es que la criminalización de las sexualidades indígenas no era sólo un acto de evangelización, sino que servía para profundizar el despojo político. Enmarcar a los pueblos indígenas como sodomitas legitimó la apropiación de sus territorios bajo la Doctrina del Descubrimiento. Esta doctrina cristiana se basaba en la noción de terra nullius, o tierra de nadie, establecida en una bula papal en 1095 por el Papa Urbano II. La bula del siglo XI legitimaba las cruzadas en Palestina: consideraba que las tierras ocupadas por "naciones bárbaras" eran terrenos "baldíos" y alentaba a los cristianos a invadirlos para dominar a su pueblo y someter esos territorios a la fe cristiana (Newcomb, 2008). En 1493, el Papa Alejandro VI reciclo este concepto de las cruzadas y emitió cuatro bulas que establecían una "ley de las naciones" cristiana que alentaba la invasión del Nuevo Mundo (Miller, 2011). Las bulas papales Inter Caetera "concedieron" a España y Portugal el derecho de conquistar las tierras descubiertas en América si no estaban bajo la fe cristiana. Al igual que Palestina, las Américas podían ser invadidas en una "guerra justa" para forzar sus habitantes a obedecer a la orden cristiana. La noción de terra nullius estableció la fe cristiana como la única fuente de autoridad legítima, definiendo los territorios indígenas como tierras baldías que las monarquías europeas se podían apropiar.

Este principio colonial de superioridad europea se convirtió en un fundamento del derecho internacional (Anghie, 2007). Sin embargo, esta doctrina sólo podía funcionar si los pueblos indígenas eran enmarcados como "bárbaros" o "salvajes" que necesitaban "ser llevados a la fe", si eran "todos sodomitas" en el sentido de desobedecer al orden divino. Fue asi que el concepto de sodomía fue usado de manera frecuente para asentar las bases de la invasión colonial, definiendo el despojo como descubrimiento y las poblaciones no europeas como ocupantes incompetentes (incivilizados) que necesitan un tutor. Dicho de otra manera, la sodomia permitió lo que Vine Deloria (2006) llamó una conquista disfrazada de ley. ${ }^{3}$

Las crónicas coloniales describían repetidamente a los nativos como sodomitas para servir los intereses territoriales de las monarquías europeas. No tenían la intención de describir las prácticas o sociedades indígenas, sino que definirlos como ilegítimos para enmarcar la guerra justa contra los pecadores fuera de la fe cristiana. Los invasores europeos beneficiaban al reportar a los pueblos del Nuevo Mundo como sodomitas, e sus reportes estaban marcados por excesos y extrapolaciones, haciendo uso abundante de los términos más

3 En su libro Dios es rojo (1972), Vine Deloria analiza el lenguaje utilizado por el Papa Alejandro VI en la bula de 1493 . 
ambiguos como sodomía, crímenes contra natura y pecados abominables. Los colonizadores enmarcaban sus relatos en el marco legal de la doctrina del descubrimiento para justificar la invasión.

Acusaciones de sodomía separaron los cuerpos "civilizados" de los cuerpos "salvajes" durante siglos. La obsesión jurídica con la sodomía se intensificó en el siglo XVIII. En una investigación archivística de 327 documentos de Nueva España, Zeb Tortorici (2018: 9) encontró más de 170, son del siglo XVIII, 80 de las dos primeras décadas del siglo XIX, un poco más de 50 casos del siglo XVII y sólo 13 del XVI. Las leyes de sodomía se fueron desmantelando de manera tan arbitraria como fueron creadas. La Revolución Francesa despenalizó la homosexualidad en Francia, pero no en Haití y la mayoría de las antiguas colonias del Imperio Británico mantuvieron las leyes de sodomía, especialmente en Uganda, que criminalizaron aún más las prácticas masculinas del mismo sexo. En la India, la Sección 377 del Código Penal, introducida en 1861, seguía vigente en 2018. En las Américas, países como Brasil y Guatemala anularon las leyes sobre "delitos contra natura" durante las reformas liberales de finales del siglo XIX, pero Nicaragua mantuvo esa legislación a través de la revolución sandinista hasta que la reforma constitucional de 2009 la abolió.

La colonización no despoja solamente sexualidades sino que también la estructura familiar indigena. Estevão Fernandes y Barbara Arisi (2017) explican que la colonización impuso una configuración europea de las relaciones íntimas con la familia nuclear a través de estructuras burocráticas para civilizar a los indios en Brasil. Circa 1750, el Directorio de Indios estableció un control administrativo de la intimidad y la domesticidad que reestructuró el sexo y el género en la vida cotidiana. Las intervenciones burocráticas se enfocavan en la heterosexualidad obligatoria, denunciando la "incivilidad" de los hogares indígenas donde "varias familias (...) viven como bestias que no siguen las leyes de la honestidad (...) debido a la diversidad de los sexos" (Fernandes; Arisi, 2017: 32). Los hogares indígenas estaban sujetos a las monógamas "leyes de honestidad" y la heterosexualización marcava el a inició el proceso de civilización de los indios. Rifkin (2011: 9) se refiere a un proceso similar en Norte América como "heterohomemización", home significando hogar en ingles. Los puntos de vista de los cristianos colonizadores coloniales categorizados como pueblos y formas de vida "sin iglesia" que existen fuera de la binaria de género (Simpson, 2017). La heteronormatividad hizo imposible que existiera cualquier otra sexualidad, género u organización familiar que no sea el modelo heterosexual de familiar nuclear europea. Los pueblos indígenas se vieron obligados a traducirse en términos autorizados por el régimen colonial y su jurisdicción. 
Este colonizar intimo no fue una especificidad de Brasil, sino que una matriz colonial. La colonización sexual siempre tuvo la misma intención colonial: destruir para reemplazar (Wolfe, 2006). Leanne B. Simpson plantea este argumento en el caso del colonialismo canadiense sobre su pueblo, ya que ese Estado se apropió de los derechos "legales" sobre las tierras Nishnaabeg para colonizar y extraer recursos naturales. Para ella, el despojo y la eliminación de los cuerpos Nishnaabeg de sus territorios facilitan el extractivismo y la autoridad colonial porque no sólo elimina la resistencia física, sino que "también borra las relaciones políticas que se encuentran dentro de los cuerpos indígenas y que vinculan nuestros cuerpos con nuestra tierra” (Simpson, 2017: 42). Así como las acusaciones de sodomía se usaban para despojar y masacrar, los pueblos indígenas fueron obligados a conformarse con la matriz de familia heterosexual colonial para sobrevivir. La heterosexualización sirvió como herramienta de homogeneización política, de dominación colonial. Además de ofuscar las diversas y complejas sexualidades indígenas, esto fue un proyecto de destrucción de las ontologías indígenas, de su comprensión del cosmos y desaparición de sus sistemas de gobierno.

\section{Relaciones decoloniales}

El deseo mismo fue colonizado, y esta colonización no terminó con las declaraciones de independencia. Para Ngũgĩ wa Thiongo (1986), la colonización de la mente está incrustada en una colonización del deseo, y a esto también se refiere Leanne B. Simpson cuando habla de un despojo expansivo que funciona a través de la violencia mas intima. Sin embargo, no debemos asumir que el despojo logro ser completo como lo asumen los regímenes coloniales. Sigal (2011) argumenta que el proceso de asimilación cultural (y sexual) fue mucho más fragmentado de lo que se supone, y que de hecho sigue incompleto. El cuerpo - con la sexualidad - contribuye una perspectiva crucial para pensar de manera critica el sistema colonial y consolidar procesos de libre determinación entre pueblos.

Siglos de colonización sexual lograron desplazar nociones originarias sobre el cuerpo y la sexualidad, difundiendo la homofobia dentro de las comunidades indígenas. Para eso, los pueblos indígenas enmarcan sus reivindicaciones dentro del marco internacional de derechos LGBT y piden el matrimonio igualitario. En 2013, la Comisión Interamericana de Derechos Humanos escuchó testimonios en un panel sobre la "Situación de los derechos humanos de las personas indígenas lesbianas, gays, bisexuales, transexuales e intersexuales en las Américas". En los Estados Unidos, al menos tres naciones indígenas han 
reconocido formalmente la igualdad en el matrimonio para las parejas del mismo sexo en sus territorios. En Brasil, el Encuentro Nacional de Estudiantes Indígenas planteó la cuestión de los derechos LGBT desde 2017, discutiendo la libre determinación desde la demarcación de tierras hasta temas de libertad sexual.

Las sexualidades indígenas vibran en las periferias donde los procesos coloniales son mas incompletos. Es el caso de Benjamin Constant, una ciudad en las periferias mas lejanas de la Amazonia en la triple frontera entre Brasil, Perú y Colombia, situada en el valle del río Javari, región con mas pueblos indígenas en aislamiento voluntario y accesible solo por bote. El pueblo cuenta con un equipo queer de fútbol desde 2002 y un bloque de carnaval gay llamado Las Marías. En 2011, Benjamín Constant celebró su primer Orgullo Gay, con mujeres trans bailando el himno gay "Sobreviviré". Una de las participantes fue la profesora de matemáticas de la escuela secundaria de la ciudad vecina Atalaia do Norte. Silvana vive su vida personal como mujer pero trabaja como hombre, comparte una casa con su marido y es aceptada como Silvana por los vecinos; pero todas las mañanas vuelve a su ser masculino para enseñar como hombre en la escuela.

Están utilizando la legitimidad internacional de las normas LGBT para reivindicar los derechos sexuales y visibilidad en sus propios términos. Sin embargo, el resurgimiento de las sexualidades indígenas no tiene que ver con el sexo o el cuerpo, sino con procesos mas profundos de libre determinación. Descolonizar las sexualidades indígenas significa habilitar mundos y formas de vida indígenas, cosmovivencias intraducibles. Simpson (2017) ve este proceso decolonial interno como un rechazo de la autoridad, dominación y heteropatriarcado coloniales, un rechazo que genera la libre determinación: un rechazo generativo.

Se puede ver un proceso de re/generación de relaciones en el pueblo Māori. Los conceptos tradicionales Māori sobre la sexualidad eran muy diferentes de lo que son hoy en día en Aotearoa/Nueva Zelanda (Te Awekotuku, 1996). La sexualidad era una parte flexible de la propia identidad, que podía evolucionar con la edad, la ubicación y la participación política (Khayatt, 2002). Sin embargo, la colonización sexual permeó la sociedad Māori, que ahora replica normas binarias de género, la homofobia, y la noción de pecado (Fergusson et al., 2005). Algo de cosmovivencia Māori resiste, ${ }^{4}$ como la idea de kaupapa, una forma única Māori de ver el mundo (Aspin; Hutchings, 2007). Existen evidencias de que la aceptación de la diversidad sexual que existía en la sociedad Māori preeuropea sigue existiendo. 
En Māori, takatāpui describe las amistades íntimas entre personas del mismo sexo. Desde la década de 1980, takatāpui se utiliza cada vez más junto con términos ingleses como gay y queer. Ahora, el término Māori se populariza. David Murray (2003) relaciona la creciente popularidad del término con la influencia combinada de tres movimientos durante ese período de tiempo: El activismo LGBT, el VIH/SIDA y el renacimiento político y cultural de Māori. Sostiene que la combinación de estos tres factores hizo posible el surgimiento de takatāpui, enfatizando la contingencia histórica y la política que condujo a la producción lingüística y cultural. El activismo gay marcó una nueva era, pero muchos individuos de Māori estaban más preocupados por combatir las injusticias sociales dentro de sus comunidades indígenas. La crisis del VIH/SIDA golpeó al pueblo Māori. La organización Māori Te Ropu Tautoko se basó en grupos regionales que se enfocaron en takatāpui, señalando la nueva instrumentalidad del término en la lucha contra el SIDA. La aprobación de la Ley del Idioma Māori en 1987 fue crucial para que Te Reo Māori se convirtiera en un idioma oficial vivo utilizado en los tribunales y en las esferas gubernamentales. El resurgimiento de takatāpui es el resultado de las complejas relaciones entre la sexualidad, la lengua y la identidad indígena de Māori. Las subjetividades sexuales están ligadas al lenguaje, y el lenguaje está conectado a las contingencias históricas. Esta defensa de los conocimientos del pasado para negociar la política actual permite a los pueblos de Māori definir su mundo por sí mismos. Están, como sugieren Aspin y Hutchings (2007), revindicando el pasado para dar forma a su futuro.

Las mujeres tikunas en la Amazonia brasilera también tiene estrategias de rechazo generativo. Ancestralmente, el pueblo Tikuna establece parejas según el clan, no al genero (Picq; Tikuna, 2016). La sociedad Tikuna se basa en su Ley de Naciones para autorizar matrimonios entre clanes según reglas de exogamia. Casarse bien es casarse con personas de diferentes clanes: un miembro del clan del pájaro (ewi) puede casarse con un miembro del clan del jaguar (ai), pero no con un miembro de su propio clan. Las uniones dentro de un clan son consideradas incestuosas. Pero las cosas empezaron a cambiar con la reciente llegada de los grupos evangélicos. Las iglesias neopentacostales recién llegadas han introducido nueva remplazando las reglas del clan por reglas de género. Estas iglesias enmarcaron las relaciones homoafectivas como pecado, e imponen una retórica religiosa que define relaciones entre mujeres de lesbianas, prohibidas y contra natura.

Para las mujeres tikunas, la noción de 'amores prohibidos' es una interferencia cristiana. "No puede estar equivocado, si lo estuviera debería haber sido 
desde el principio y no algo nuevo. (...) Nuestros antepasados tenían personas viviendo vidas homoafectivas pero nunca lo interpretaron como algo malicioso, es la religión la que vino a interferir con nuestra cultura tratando de evangelizarnos" (Picq; Tikuna, 2016). En un acto de resurgimiento indígena, las mujeres tikunas revindican la Ley de Naciones para defender su autonomía para amar en términos tikunas. Defienden la relación homo-afectiva como consistente con reglas ancestrales de exogamia del clan. Para ellas, no hay duda de que las codificaciones sexuales fueron introducidas por las Iglesias evangélicas, e por eso resisten la autoridad misionera sobre sus cuerpos y sus familias. Están construyendo sus propias familias para mostrar que las relaciones homoafectivas no interfieren de ninguna manera con las visiones del mundo tikuna, al contrario, se complementan entre sí. Como el pueblo Māori, están reclamando el pasado para dar forma a un futuro decolonial que respete la libre determinación.

Para la profesora, autora y activista Nishnaabeg Leanne B. Simpson (2017), la familia indígena encarna formas alternativas de gobierno que se niegan a replicar el capitalismo y la heteropatracia del estado colonial. Las familias y sexualidades indígenas son vistas como peligrosas porque reproducen y amplifican el mundo indígena (Simpson, 2017: 41). Las familias indígenas fueron criminalizadas como contra natura, disfuncionales y peligrosas precisamente porque reproducían la libre determinación y desafiaban estructuras heteropatriarcales de autoridad. En Canadá y en Estados Unidos, miles de niños indígenas fueron robados de sus familias y secuestrados en internados que operaban como cárceles con el propósito de destruir las redes familiares, comunitarias, lingüísticas, separando familias, rompiendo vínculos intergeneracional de saberes, y aislando cuerpos de sus territorios. La política estatal era "matar el indio para salvar el hombre".

La familia indígena es un espacio privilegiado para reverter el despojo colonial por que existe en oposición directa al modelo familiar heteropatriarcal como espacios de autonomía que producen relaciones intergeneracionales de libre determinación. Desafían la autoridad patriarcal característica de la familia nuclear colonial y valoran el consentimiento como fundamento para aprender y relacionarse (Morgensen, 2011: 4). En la cosmovisión de Yoate, por ejemplo, se considera que los bebés eligen a la familia donde nascen (Tallbear, 2018: 150) los niños entran a las familias por su propia voluntad, y por lo tanto nacen con consentimiento. Las familias continúan nutriendo este consentimiento innato al incluir al niño como un miembro integral de la familia, capaz de tomar decisiones y tener responsabilidades. Así mismo, los pueblos Nanibush y Kappo consideran que los niños tienen que ser parte de la toma de decisiones para la 
supervivencia futura de sus comunidades. Para ellos, el rol de los niños como actores de decisión es un elemento clave del restablecimiento de la gobernanza tradicional (Kuokkanen, 2019: 223). Hay cosmovisiones que valoran a los niños igual que los abuelos porque consideran el aprendizaje como un proceso infinito y no lineal: "No puedes graduarte en Nishnaabemwin; es un regalo que debe ser practicado y reproducido", dice Simpson (2017: 154). Los adultos no son intrínsecamente las autoridades más conocedoras o capaces en todas las situaciones, y deben pedir ayuda, ya sea a otros adultos o niños. Los niños pueden nacer con dones únicos con los que otros no nacen, y sus formas únicas de conocer los mundos son alentadas, valoradas y confiadas.

En estos contextos, el concepto de maternidad es profundamente distinto del modelo occidental que pone la responsabilidad de criar a los hijos en la madre. La noción de madre soltera ni siquiera existe en la mayoría de las cosmovisiones indígenas, "porque las mujeres nativas y sus hijos vivían y trabajaban en redes de parentesco extendidas" (Tallbear, 2018: 150). Jeff Corntassell y Mick Skow (2017) describen redes de amigos que actúan como "otras madres" y "otros padres" para sus niños cuando los parientes biológicos viven lejos. La noción de madre soltera aparece con la destrucción de las familias indígenas amplias, las separa de su territorialidad, suprimiendo todas sus relaciones. En el contexto Māori, por ejemplo, el despojo de tierras destruyó grandes redes de parentesco comunal y obligó los pueblos a habitar en familias nucleares más pequeñas. Este despojo colonial erosionó métodos Māori de criar a los niños: "a principios del siglo XX, no todos los Māori tenían acceso a la tierra, y no estaban en la posición afortunada de poder tener una vida extendida en un hogar familiar comunal" (Connor, 2014: 235).

\section{Conclusión}

Ya sea a través del lenguaje, la cerámica o la ceremonia, se pueden encontrar ejemplos de diversidad sexual en cantidad de sociedades indígenas, desde la época precolombina hasta la actualidad. La diversidad de referentes y prácticas de género han sido ampliamente documentadas en diferentes momentos históricos y en muchas culturas. Los significados sociales y políticos difieren profundamente según el contexto, y muchas veces son imposibles de traducir. La cuestión no es si la diversidad sexual existe entre los pueblos indígenas, sino cómo sigue resistiendo siglos de despojo y asimilación heteronomartiva.

Una conclusión es que las sexualidades diversas y los géneros múltiples no son una introducción occidental y reciente en los mundos indígenas. Lo que fue 
importado de Europa no es la parada del orgullo gay sino que la heterosexualidad. La colonización sexual fue una herramienta de disciplina para controlar a los pueblos de Abya Yala e una herramienta de conquista para justificar la doctrina del descubrimiento. Las relacionalidades indígenas fueron reprimidas, patologizadas y borradas por procesos violentos de despojo colonial. La resistencia de estas sexualidades es prueba de que existen pedazos de mundo que sobrevivieron el inmenso proceso de colonización. Las sexualidades indígenas son importantes sitios de resistencia y libre determinación. Resisten al colonialismo heteronormativo; encarnan la posibilidad de un reexistir radical. Las sexualidades indígenas importan más allá de la política sexual porque expanden la imaginación política, abriendo la posibilidad de otras formas de relacionarnos entre seres en el mundo.

\section{Referências}

ALFRED, Taiaiake; CORNTASSEL, Jeff. Being Indigenous: Resurgences Against Contemporary Colonialism. Government and Opposition, v. 40, n. 4, 2005, pp. 597-614. ANGHIE, Antony. Imperialism, Sovereignty and the Making of International Law. Cambridge, Cambridge University Press, 2007.

ASPIN, Clive; Hutchings, Jessica. Reclaiming the past to inform the future: Contemporary views of Maori sexuality. Culture, Health \& Sexuality, v. 9, n. 4, 2007, pp. 415-427.

ARVIN, Maile; TUCK, Eva; MORRILL, Angie. Decolonizing Feminism: Challenging Connections between Settler Colonialism and Heteropatriarchy. Feminist Formations, v. 25, n. 1 (Spring) 2013, pp. 8-34.

BLACKWOOD, Evelyn. Sexuality and gender in certain Native American tribes: The case of cross gender females. Signs, v. 10, n. 1, 1984, pp. $27-42$.

CARVAJAL, Federico. Butterflies will burn: Prosecuting Sodomites in Early Modern Spain and Mexico. Austin, University of Texas Press, 2003.

CARILLO CAN, Isaac E. El erotismo andróginx en la cosmovisión y lenguaje Maya. Andar Erótico Decolonial. Buenos Aires, Ediciones del Signos, 2015, pp. 73-81.

CONNOR, Helene. Māori Mothering: Repression, Resistance and Renaissance. In: Mothers of the Nations: Indigenous Mothering as Global Resistance, Reclaiming and Recovery. Indigenous Mothering as Global Resistance, Reclaiming and Recovery, 2014, pp. 231-50.

CORNTASSEL, Jeff; SKOW, Mick. Everyday Acts of Resurgence: Indigenous Approaches to Everydayness in Fatherhood. New Diversities, v. 19, n. 2, 2017, pp. 55-68.

COTTET, Caroline; PICQ, Manuela. Sexuality and Translation in World Politics. S.l., E-International Relations, 2019. 
DELORIA, Vine Jr. Conquest Masquerading as Law. In: Unlearning the Language of Conquest: Scholars Expose Anti-Indianism in America, edited by Four Arrows. Austin, University of Texas Press, 2006, pp. 94-107.

DRISKILL, Qwo-Li, ed. Queer Indigenous studies: Critical interventions in theory, politics, and literature. Tucson, University of Arizona Press, 2011.

FERGUSSON, David M.; HORWOOD, L. John; RIDDER, Elizabeth M.; BEAUTRAIS, Annette Louise. Sexual Orientation and Mental Health in a Birth Cohort of Young Adults. Psychological Medicine, v. 35, n. 7, 2005, pp. 971-81.

FERNANDES, Estevão Rafael; ARISI, Barbara M. Gay Indians in Brazil. Springer, 2017. HAWKINS, Philip Colin. New World Sodom: Biblical Tales of Conquest and Acculturation. Electronic Journal of Human Sexuality, v. 15, 2012.

HORSWELL, Michael J. Decolonizing the Sodomite: Queer Tropes of Sexuality in Colonial Andean Culture. Austin, University of Texas Press, 2005.

HUMAN Rights Watch. This Alien Legacy: The Origins of Sodomy Laws in British Colonialism. 2008. Disponível em: <www.hrw.org/sites/default/files/reports/ lgbt1208_webwcover.pdf >. Acesso em: 03 abr. 2020.

JORDAN, Mark D. The Invention of Sodomy in Christian Theology. Chicago, University of Chicago Press, 1998.

KATZ, Jonathan N. The Invention of Heterosexuality. Chicago, Chicago University Press, 2007. KAUANUI, Kehaulani. Hawaiian Blood: Colonialism and the Politics of Sovereignty and Indigeneity. Durham, Duke University Press, 2008.

KHAYATT, Didi. Towrads a Queer Identity. Sexualities, v.5, n. 4, 2002, pp. 487-501.

KIMBALL, Geoffrey. Aztec homosexuality: the textual evidence. Journal of Homosexuality, v. 26, n. 1, 1993, pp. 7-24.

KUOKKANEN, Rauna. Restructuring Relations: Indigenous Self Determination, Governance and Gender. New York, Oxford University Press, 2019.

MOLINA, Fernanda. Cuando amar era pecado: Sexualidad, poder e identidad entre los sodomitas coloniales. Lima/LaPaz, Plural Editores, 2017.

MILLER, Robert J. The International Law of Colonialism: A Comparative Analysis. Lewis \& Clark Law Review, Research Paper, n. 2011-23, 2011.

MIRANDÉ, Alfredo. Behind the Mask: Gender Hybridity in a Zapotec Community. Tucson, University of Arizona Press, 2017.

MORGENSEN, Scott Lauria. Spaces between us: Queer settler colonialism and indigenous decolonization. Mineápolis, University of Minnesota Press, 2011.

MURRAY, David AB. Who is Takatāpui? Māori language, sexuality and identity in Aotearoa/New Zealand. Anthropologica, 2003, pp. 233-244.

NAJMABADI, Afsaneh. Women with Mustaches and Men without Beards: Gender and Sexual Anxieties of Iranian Modernity. California, University of California Press, 2005. 
NEWCOMB, Steve. Pagans in the Promised Land: Decoding the Doctrine of Christian Discovery. Fulcrum, 2008.

NGŨGĨ WA THIONG'O. Decolonizing the Mind: The Politics of Language in African Literature. Melton, Reino Unido, James Currey, 1986.

OLITA, Ivan. Third Gender: An Entrancing Look at México's Muxes. Artistic Short Film. National Geographic, Short Film Showcase, February 2017.

PUPO-WALKER, Enrique (Ed.). Castaways: The Narrative of Alvar Nuñez Cabeza de Vaca. California, University of California Press, 1993.

PURI, Jyoti. Sexual States: Governance and the Struggle Over the Antisodomy Law in India. Durham, Duke University Press, 2016.

RIFKIN, Mark. When did Indians become straight? Kinship, the history of sexuality, and native sovereignty. Oxônia, Reino Unido, Oxford University Press, 2011.

ROBERTSON, Carol E. The mahu of Hawai'i (an art essay). Feminist Studies, v. 15, n. 2, 1989, p. 313.

ROSCOE, Will. Changing Ones: Third and Fourth Genders in Native North America. Londres, Palgrave, 1998.

RUVALCABA, Héctor Domínguez. Translating the Queer: Body Politics and Transnational Conversations. Londres, Zed Books, 2016.

SETH, Vanita. Europe's Indians: Producing Racial Difference, 1500-190o. Durham, Duke University Press, 2010.

SIGAL, Pete. The flower and the scorpion: Sexuality and ritual in early Nahua Culture. Durham, Duke University Press, 2011.

SIMPSON, Leanne Betasamosake. As We Have Always Done: Indigenous Freedom Through Radical Resistance. Minneapolis, University of Minnesota Press, 2017.

SPIVAK, Gayatri C. The politics of Translation" in Outside in the Teachign Mahcine. New York, Routledge, 1993, pp. 179-200.

SVATI P. Shah. 2019. Sedition, Sexuality, Gender, and Gender Identity in South Asia. South Asia Multidisciplinary Academic Journal [Online], $20 \mid$ 2019, Online connection on 25 July 2019. Disponível em: <http://journals.openedition.org/samaj/5163. Acesso em: 07 abr. 2020.

TALLBEAR, Kim. Making Love and Relations Beyond Settler Sex and Family. In: Making Kin Not Population. Chicago, Prickly Paradigm Press, 2018, pp. 145-64.

TE AWEKOTUKU, Ngahuia. He Reka Ano: same sex lust and loving in the ancient Maori world. Outlines: Lesbian \& Gay Histories of Aotearoa. Wellington, Lesbian \& Gay Archives of New Zealand, 2005.

TE AWEKOTUKU, Ngahuia. "Maori: People and Culture". Maori Art and Culture, 1996, pp. 114-46.

TENGAN, Ty P. Kāwika. Native Men Remade: Gender and Nation in Contemporary Hawai'i. Durham, Duke University Press, 2008. 
TIKUNA, Josi; PICQ, Manuela. Queering Amazonia: Homo-Affective Relations among Tikuna Society. Queering Narratives of Modernity. Peter Lang/FLACSO, 2016, pp. 113-134.

TORTORICI, Zeb. Sins Against Nature; Sex \& Archives in Colonial New Spain. Durham, Duke University Press, 2018.

TREXLER, Richard C. Sex and conquest: Gendered violence, Political Order, and the European Conquest of the Americas. Ithaca, Nova York, Cornell University Press, 1999.

VAN DEUSEN, Nancy. Global Indios: The Indigenous Struggle for Justice in SixteenthCentury Spain. Durham, Duke University Press, 2015.

WEISMANTEL, Mary. Moche Sex Pots: reproduction and temporality in ancient South America. American Anthropologist, v. 106, n. 3, 2004, pp. 495-505.

WILSON, Joe; HAMER, Dean. Kumu Ina. Documentary film by Independent Lens, 2014. WOLF, Eric. Europe and the People Without History. California, University of California Press, 1982.

WOLFE, Patrick. Settler Colonialism and the Elimination of the Native. Journal of Genocide Research, v. 8, n. 4, 2006, pp. 387-409.

Recebido em: 04/02/2020

Aprovado em: 20/03/2020

\section{Como citar este artigo:}

PICQ, Manuela L. La colonización de sexualidades indígenas: entre despojo y resistencia. Contemporânea - Revista de Sociologia da UFSCar, v. 10, n. 1, jan.- abril 2020, pp. $13-34$ 